\title{
Premotor Cortex Neurons in Macaques: Activity Before Distal and Proximal Forelimb Movements
}

\author{
Kiyoshi Kurata ${ }^{1}$ and Jun Tanji \\ Department of Physiology, School of Medicine, Hokkaido University, Sapporo, 060, Japan
}

\begin{abstract}
Single unit activity was examined in the premotor cortex of three awake, behaving macaque monkeys. Premotor cortex (PM) neurons were found to be active in association with a movement involving specifically the distal forelimb. Other PM neurons were active in relation to more proximal forelimb movements. The spatial distribution of neurons related to distal movements differed from that of neurons related to proximal forelimb movements, the former being focused at a postarcuate region near the genu of the arcuate sulcus and the latter being shifted more posteromedially. The distal movement was triggered by one of the three sensory signals: visual, auditory, and vibrotactile. Of $190 \mathrm{PM}$ neurons related to the distal forelimb movements, 123 $(65 \%)$ showed similar premovement activity changes irrespective of the modality of the triggering signal. However, a number of neurons (35\%) responded preferentially to one or two of the three signals. The modality-specific activity before movements distinguishes PM from the caudally adjacent primary motor cortex.
\end{abstract}

Numerous concepts have developed concerning the functional organization of a motor field in the frontal agranular cortex rostral to the precentral (or primary) motor cortex and lateral to the supplementary motor cortex (for review, see Humphrey, 1979; Wiesendanger, 1981; Wise, 1985). We will refer to this area as the premotor cortex (PM), following a proposal recently made by Weinrich and Wise (1982; see also Sessle and Wiesendanger, 1982; Wise, 1985). It should be noted that this area, or parts of it, has also been referred to as area 6 (Brodmann, 1909), area 6aß. (Humphrey, 1979; Vogt and Vogt, 1919), postarcuate area (Godschalk et al., 1981; Rizzolatti et al., 1981a, b), or areas FB and FC (Bonin and Bailey, 1947; Kubota and Hamada, 1978).

There are at least two different views regarding the functional role of the PM. On one hand, on the basis of anatomical studies of descending fiber connections (Kuypers and Brinkman, 1970; Kuypers and Lawrence, 1967) and clinical observations (Freund and Hummelsheim, 1984), the PM has been considered to control exclusively or mainly the proximal limb or axial musculature. The results from electrical stimulation studies, in which no distal limb movements could be evoked from this area (Woolsey et al., 1952), have been taken to support this view. On the other hand, the PM has been viewed as an area controlling skilled movements of the whole body (Fulton, 1934; see

Received Oct. 16, 1984; revised May 6, June 30, 1985; accepted July 2, 1985.

Dr. M. E. Anderson, Department of Rehabilitation Medicine, University of Washington, Seattle, participated in the early part of this study. We thank Professor M. Kato for his generous support and advice on the manuscript, Miss Y. Kobayashi for excellent technical assistance, and Mrs. K. Amano for typing the manuscript. This work was supported in part by Ministry of Education, Science and Culture of Japan Grants 58106001 and 58570046

Correspondence should be addressed to Dr. Tanji.

Present address: Laboratory of Neurophysiology, BIdg. 36, Rm. 2D-10, NIMH, NIH, Bethesda, MD 20205.

Copyright (C) 1986 Society for Neuroscience $0270-6474 / 86 / 020403-09 \$ 02.00 / 0$ also Wiesendanger, 1981) or as having a specific role in sensory guidance of movements (Godschalk et al., 1981; Halsband and Passingham, 1982; Rizzolatti et al., 1981b; see also Moll and Kuypers, 1977). This view is supported by anatomical studies demonstrating corticocortical afferents to PM from visual, auditory, and somatosensory association cortex (Chavis and Pandya, 1976; Pandya and Kuypers, 1969; Petrides and Pandya, 1984), as well as topographically organized efferent connections to the motor cortex (Muakkassa and Strick, 1979; see also Godschalk et al., 1984; Matsumura and Kubota, 1979).

One issue central to testing the validity of these two theories is the presence or absence of neuronal activity related exclusively to distal limb movements. Recent single unit studies in trained monkeys suggest a participation of PM neurons in distal as well as proximal forelimb movements. Kubata and Hamada (1978) reported PM neuronal activity in relation to a handle manipulation movement performed mainly with wrist extension and flexion. However, since the forearm was only loosely restrained, there is the possibility that the neuronal activity was actually related to proximal forelimb movements, such as postural fixation movements involving shoulder or axial musculature, rather than wrist movements. In a recent review article, Brinkman and Porter (1983) reported preliminary observations on PM neurons that seemed to be related to distal forelimb movements. However, when the animal is freely moving with its forelimbs unrestrained, as in their experimental design, any distal forelimb movements should inevitably be accompanied by supporting activity in proximal limb and axial muscles. Thus, an unequivocal demonstration of PM neuronal activity as exclusively related to distal forelimb movements is still lacking. In an attempt to resolve this question, the present series of experiments was specifically designed to compare the neuronal activity associated with a distal and a proximal forelimb movement.

The second aim of the present study was to compare the PM neuronal activity in response to signals of three different modalities serving as a trigger to start the movement. The importance of visual inputs to the PM in initiating or guiding movements has been indicated by lesion studies (Haaxma and Kuypers, 1975; Halsband and Passingham, 1982; Moll and Kuypers, 1977) and single unit recording studies (Godschalk et al., 1981; Kubota and Hamada, 1978; Rizzolatti et al., $1981 \mathrm{~b}$; Weinrich and Wise, 1982); as well as anatomical evidence that the $P M$ receives afferents from sensory association areas (Chavis and Pandya, 1976; Godschalk et al., 1984; Pandya and Kuypers, 1969; Petrides and Pandya, 1984). The present study cxamined the relative potency of three sensory inputs-one visual, one auditory, and one somatosensory-in activating PM neurons prior to movement initiation.

\section{Materials and Methods}

Behavioral paradigm

Three male Japanese monkeys ( Macaca fuscata) were used in the present experiments. They were cared for in the manner prescribed in Guiding 
midline

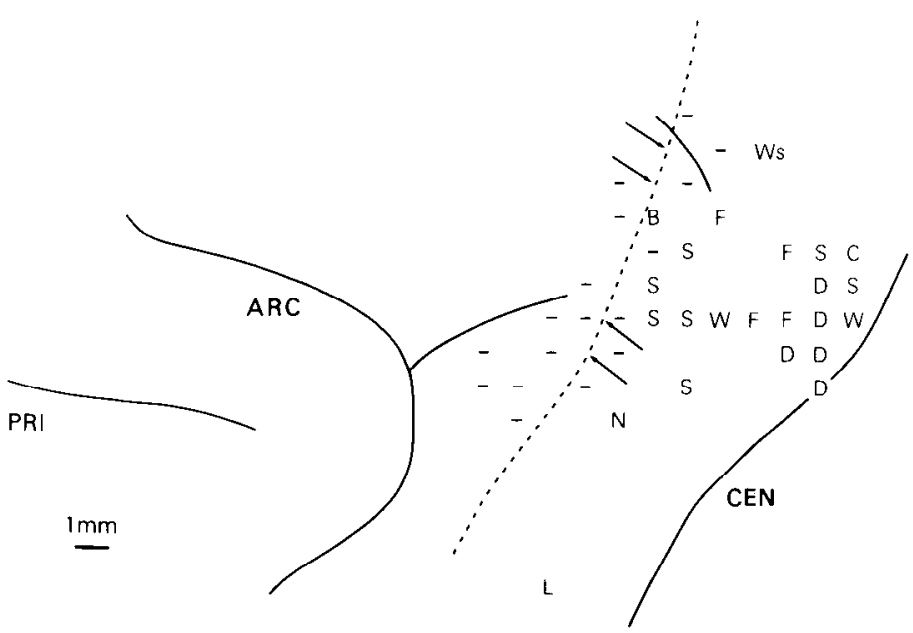

Figure 1. Surface reconstruction of intracortical microstimulation results and the location of the cytoarchitectonic boundary in the left hemisphere. Positive sites for evoking movements at stimulus intensity of less than $50 \mu \mathrm{A}$ are indicated by letters: $D$, digits; $W$, wrist; $F$, forearm; $S$, shoulder; $C$, chest; $B$, back; $W S$, waist; $L$, lips; $N$, Neck. Negative sites are indicated by short horizontal bars. The cytoarchitectonic boundary was determined by counting giant layer $\mathrm{V}$ pyramidal cells and is indicated by an interrupted line. The arrows indicate sites where quantitative studies were done as described in the text. The abbreviations for sulci are $C E N$, central sulcus; $A R C$, arcuate sulcus; $P R I$, principal sulcus.

Principles in the Care and Use of Animals of the American Physiological Society. Each monkey was seated in a primate chair and trained to perform separately execuled distal or proximal forelimb movements. Both upper extremities were rigidly fixated on L-shaped plastic casts with the elbow flexed at $90^{\circ}$. The right forelimb cast was attached to a shaft and a pivot so that only inner and outer rotation at the shoulder joint could be performed. Movements of the left shoulder joint were prevented by another cast. A key was attached to the distal end of the cast to be pressed by flexion of fingers and wrist. The monkeys were required to move the cast by rotating the shoulder joint to come into one of two correct holding zones of $4^{\circ}$, which were separated $10^{\circ}$ outward and $30^{\circ}$ inward from the line perpendicular to the frontal plane. The acquisition of the correct zone was indicated by a green light-emitting diode (LED) placed on a panel $20 \mathrm{~cm}$ in front of the animal. After a variable holding period of $2.5-4.0 \mathrm{sec}$, the animal was required to press the key in response to either a visual, auditory, or somatosensory trigger signal. The modality of the trigger stimulus was selected in a pseudorandom schedule. The visual signal was a red light placed next to the panel. The auditory signal was a pure tone of $1 \mathrm{kHz}$ at an intensity of $18 \mathrm{~dB}$ above the background noise level. The somatosensory signal was a $40 \mathrm{~Hz}$ vibrotactile stimuli with an amplitude of $150 \mu \mathrm{m}$, generated by a DC motor and delivered to a probe placed on the dorsal surface of the forearm at a point $2 \mathrm{~cm}$ proximal to the wrist joint. The monkey had to keep holding the cast in the correct zone during the key press and for $2.5-4.0 \mathrm{sec}$ afterward in order to receive a reward of fruit juice, which prompted the animal to shift the cast to the other holding zone. All of these sequences of the motor task were controlled by a laboratory microcomputer.

\section{Data recording and processing}

After 4 months of training, a stainless steel recording chamber $(32 \times$ $45 \mathrm{~mm}$ ) was attached to the skull under aseptic conditions. The monkeys were anesthetized by ketamine hydrochloride and sodium pentobarbital. Conventional chronic single unit recording methods were employed (Evarts, 1968). Glass-insulated platinum iridium microelectrodes (with $1.5-2.5 \mathrm{~m} \Omega$ resistance) were driven by an electronic stepping microdrive (MO-951, Narishige) for extracellular recording. The same electrode was also used for intracortical microstimulation. A train of 12 cathodal pulses of $0.2 \mathrm{msec}$ duration at $333 \mathrm{~Hz}$ was applied at an intensity of less than $50 \mu \mathrm{A}$ through a constant-current stimulator.
On-line analysis of the neuronal discharge activity was made by using a laboratory computer. The discharge activity over 16 trials was aligned with either the signal or the movement onset and displayed as perievent raster displays. The time of closing of the key switch was defined as the movement onset for the key-press movement. For the shoulder-rotating movements, the first movement of the cast detected with a position transducer after the holding period was defined as the movement onset. Perievent time histograms were also constructed with bin widths ranging from 2 to $100 \mathrm{msec}$ (usually $20 \mathrm{msec}$ ). The neuronal activity was judged to be significantly related to a movement if the number of discharges during at least two consecutive bins among 32 bins preceding the movement onset deviated by more than $3 \mathrm{SD}$ of the mean value calculated during a control period of $1280-640$ msec preceding the movement.

Muscle activity was monitored from extensor and flexor muscles of fingers, wrist, and elbow and also from the deltoid and infraspinatus muscles with chronically implanted Teflon-coated silver wire electrodes. Muscle activity from supraspinatus, trapezius, pectoralis, serratus, paravertebral, and hip muscles in addition to these muscles was also monitored with surface electrodes. The EMG was full-wave rectified, converted from voltage to frequency, and analyzed in the same manner as the discharges of single neurons. AC-coupled recording of the electrooculogram (EOG) was also obtained with surface electrodes to monitor saccadic eye movements. In one monkey, a stimulating electrode was permanently implanted in the ipsilateral medullary pyramid at level P-2, L-1 of the Horsley-Clarke coordinate for identification of pyramidal tract neurons (PTNs). Their antidromic responses and latencies were confirmed by the spike collision method.

\section{Histological studies}

The monkeys were deeply anesthetized with an overdose of pentobarbital and perfused through the heart with saline followed by $10 \%$ formalin. The brain was removed from the skull after marking the location of the recording chamber and noting cortical landmarks, photographed, sectioned parasagittally at $50 \mu \mathrm{m}$ on a freezing microtome, and stained with thionin. Reconstruction of clcctrode tracks was attempted with the aid of electrolytic microlesions as reference points.

\section{Results}

\section{Cortical field determination of recording sites}

Recording sites were determined to be in either the PM or precentral motor cortex (MI) according to recently proposed criteria (Weinrich and Wise, 1982; see also Sessle and Wiesendanger, 1982).

\section{Cytoarchitectonic analysis}

Since no distinct boundary between PM and MI can be drawn on the basis of any cytoarchitectural characteristics, a boundary to divide the frontal agranular cortex into two grossly dissimilar areas was drawn on the basis of a density analysis of giant pyramidal cells in layer $\mathrm{V}$. The number of giant pyramidal cells having a diameter exceeding $29 \mu \mathrm{m}$ (this arbitrary value was chosen to make the data comparable with those of Weinrich and Wise) was counted in consecutive $50 \mu \mathrm{m}$ sagittal sections. The points in the anteroposterior axis where the density of the giant pyramidal cells (per millimeter in consecutive $100 \mu \mathrm{m}$ thickness of cortex) changed distinctly was plotted on a cortical surface map, and a smoothed line approximately connecting these points was used as a tentative boundary. The boundary is depicted as an interrupted line in a map shown in Figure 1. The mean number of the giant cells in 10 cortical slabs $(100 \mu \mathrm{m}$ in mediolateral extent and $2 \mathrm{~mm}$ in rostrocaudal extent) immediately rostral to and caudal to the border was $5.80 \pm 1.55$ and $15.30 \pm 4.45$, respectively, when measured from 7.0 to 8.0 $\mathrm{mm}$ lateral to the midline ( $p<0.001 ; t$ test, two-tailed). When measured from 12.0 to $13.0 \mathrm{~mm}$ lateral to the midline, the values were $4.70 \pm 1.89$ and $7.50 \pm 2.46$, respectively $(p<0.05)$.

\section{Microstimulation effects}

In a portion of the cortex rostral to the histologically determined border, no effects from microstimulation were observed at any 


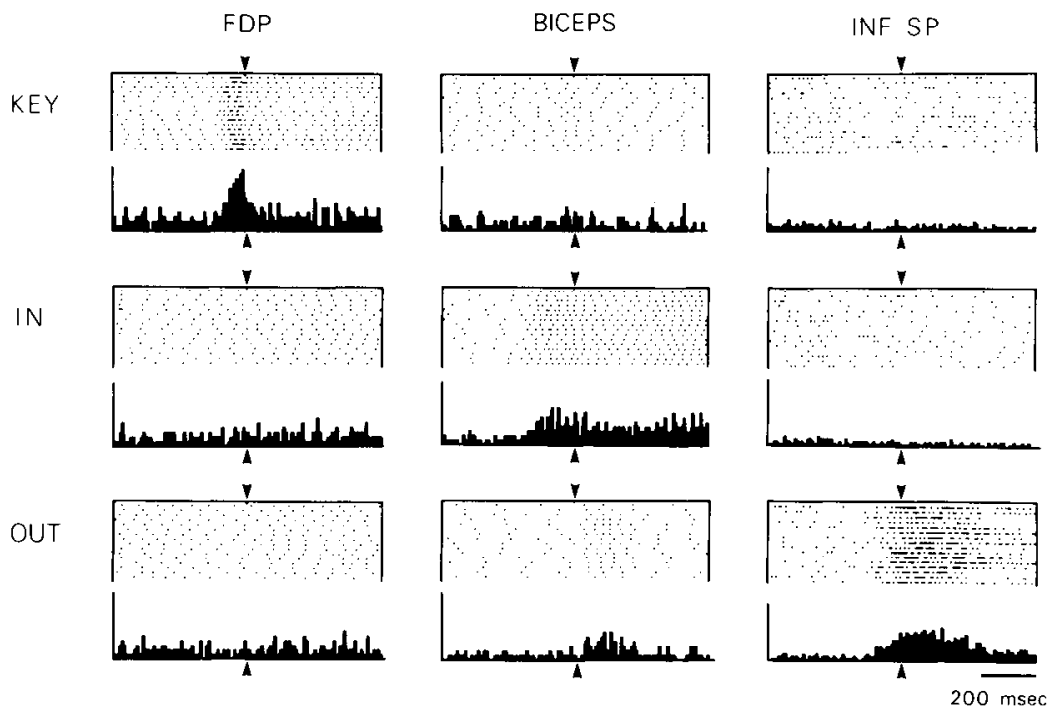

Figure 2. EMG activity in the three forelimb muscles: $F D P$, flexor digitorum profundus; $B I C E P S$, biceps brachii; $I N F . S P$, infraspinatus. EMGs are full-wave rectified, digitized, and displayed as dot rasters. Each line of the raster denotes individual trials. Results obtained in 16 trials are summed in histograms below each raster. Activities in three types of movements are indicated: (1) keypress movement by fingers and hand $(K E Y),(2)$ inward $(I N)$, and (3) outward (OUT) rotation movements around the shoulder joint. Arrowheads indicate the onset of each movement.

points of microelectrode entry (horizontal small bars in Fig. 1) with stimulus currents of less than $50 \mu \mathrm{A}$. In contrast, muscle contractions as well as limb movements were often observed caudal to the boundary (see Fig. 1). These findings agree with those of Weinrich and Wise (1982) and Sessle and Wiesendanger (1982) indicating that the anterior border of microexcitable cortex with small currents corresponds approximately with the cytoarchitectonic border. Basically similar results were obtained in the other two monkeys, and we therefore used the histologically determined border as the boundary between PM and MI.

\section{Muscle activity}

Since it was essential in this experiment to train the animal to perform isolated distal and proximal forelimb movements, extensive EMG recordings were made to examine whether selective activation of proximal and distal muscle activity was indeed obtained. In one monkey (the second of those used in the present experiments), we failed to obtain the proximal forelimb movements of shoulder rotation without concomitant activity in wrist and finger flexor muscles. Therefore, the neuronal data obtained from that monkey were excluded from the subsequent analysis except for the purpose of comparing neuronal responses to different modalities of sensory signals triggering the key-press movement (see below). In the other two monkeys, the temporal separation of proximal and distal EMG activity was complete as far as we could determine. Finger and wrist flexor muscles were phasically active prior to and during the key-press movement, but their activity was unaltered in association with inward or outward rotation movements. An example of such an EMG recorded in flexor digitorum profundus is displayed in the left column of Figure 2. In contrast, muscles such as the deltoid, supra- and infraspinatus, serratus, and pectoralis exhibited activity changes only in association with the shoulder rotation movements (right column of Fig. 2). Biceps and triceps muscles showed less activity change in association with the rotation movements and were inactive with the key-press movement. Paravertebral muscles were sometimes active during rotation movements, but the activity was variable and much weaker than those in forelimb muscles. These axial muscles showed no changes in activity in relation to the distal movements.

Saccadic cyc movements in the vertical and horizontal directions were observed during the performance of the task, but they were randomly distributed in time and not regularly timelocked with the occurrence of any sensory signals or limb movements.

\section{Neuronal activity related to the key-press and shoulder rotation movement}

Some 142 neurons recorded in the PM of the two monkeys exhibited activity changes exclusively prior to the initiation of the key-press movement and showed no activity modulation prior to the shoulder rotation movements. They will be referred to as "distal neurons." Of these 142 neurons, 48 exhibited activity changes apparently time-locked to onset of the key-press movement. Fifteen neurons showed responses time-locked to the signals that trigger the movements. In the remaining 79 neurons, the assignment of the activity changes to either type was not possible. Figure $3 A$ shows an example of movementlocked activity in a distal PM neuron, so classified because the onset of activity increase of this neuron was time-locked to the movement onset ( $\mathrm{M}$ in the right column of Fig. $3 A$ ) rather than to the signal onset (S). The amount of activity increase was similar regardless of the modality of the trigger signal. In order to test whether the neuron responds to the sensory signals even when they do not serve as a movement trigger, "extra" signals were delivered about $1 \mathrm{sec}$ after the monkey's key-press movement, to which the animal did not respond. The neuron did not show any activity increase in response to these extra signals (not shown). Additionally, in the rare instances when the monkey emitted key-press movements "spontaneously," i.e., not in response to any sensory signal, the neuron was active, as shown in the fourth row of Figure $3 A(\mathrm{NON})$. On the other hand, and most importantly, the neuron was not active in relation to the inward or outward shoulder rotation movements (SHIFT). Figure $3 A$ thus indicates an example of PM neuronal activity exclusively related to the key-press movement, regardless of whether the movement was triggered by sensory signals and regardless of which sensory signal triggered the movement. In contrast to the 142 distal neurons, 125 neurons in PM were found to change their activity only prior to the inward and/or outward rotation movement and will be called "proximal" neurons. Ten neurons showed mixed properties in that they were modulated before the proximal and distal movements in the present task. Fifteen neurons in PM were identified as pyramidal tract neurons (PTNs). These neurons were classified as distal (8), proximal (6), and mixed (1).

In the part of the cortcx defincd as MI on the basis of cytoarchitectonics and microstimulation effects, 37,71 , and 10 neurons were classified as distal, proximal, and mixed neurons. The number of task related neurons in MI obtained was small because the MI hand or finger area was not as extensively sur- 


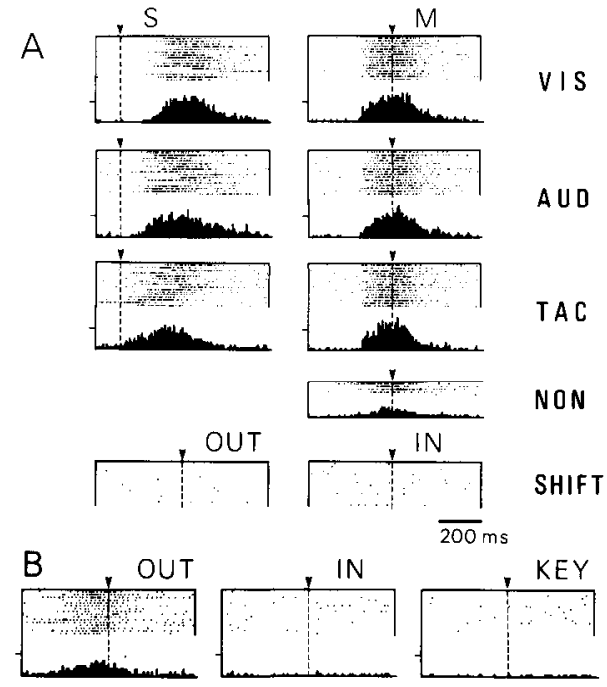

Figure 3. Discharge of two types of neurons in the premotor cortex: distal neuron $(A)$ and proximal neuron $(B)$. Neuronal discharges are indicated as dots and are aligned on the onset of signals $(S)$ or movements $(M, O U T, I N$, or $K E Y)$. In $A$, the modality of signals triggering the key-press movement is indicated to the right of raster displays: VIS, visual; $A U D$, auditory; $T A C$, vibrotactile. In the fourth row $(N O N)$, the discharge at the onset of the key-press movements not triggered by any signal is displayed. In the fifth row, the discharge at the onset of the shoulder rotation movements observed at outward (OUT) and inward $(I N)$ shift of the forearm cast is displayed. In $B$, an example of a neuron active only prior to the outward rotation movement is displayed. Tick mark on the ordinate of each histogram, $100 \mathrm{imp} / \mathrm{sec}$.

veyed as that of PM. Since the primary focus of the present study was on PM neurons, MI was not penetrated throughout its entire extent. The following data are presented for completeness but should be interpreted with caution, in view of the limited exploration of MI. Quantitative analyses were made of PM and MI neurons on (1) the amount of background activity, (2) magnitude of movement related activity, (3) the latencies of responses to sensory signals triggering the key-press movement, and (4) the duration of the neuronal activity prior to the onset of the key-press movement. The mean background discharge was $8.0 \pm 4.8 \mathrm{imp} / \mathrm{sec}$ in $P M$ and $15.6 \pm 6.7 \mathrm{imp} / \mathrm{sec}$ in MI neurons. The magnitude of the movement-related activity (the discharge frequency during $320 \mathrm{msec}$ prior to the movement onset minus the background discharge frequency) was $40.3 \pm$ 17.5 and $63.7 \pm 19.2 \mathrm{imp} / \mathrm{sec}$ in PM and MI neurons, respectively. This difference was significant at the $p<0.001$ level (Mann-Whitney $U$ test). When this analysis was limited to the movement-locked type of PM neurons, the magnitude was $40.6 \pm 10.9 \mathrm{imp} / \mathrm{sec}$, still smaller than that of MI neurons ( $p<$ 0.01 ). In PM and MI proximal neurons, the values were 29.4 \pm 11.0 and $41.2 \pm 18.1 \mathrm{imp} / \mathrm{sec}$ (not significantly different by Mann-Whitney $U$ test). The timing of neuronal activity in relation to the triggering stimuli and movement onset is described in detail later.

\section{Distribution of distal and proximal neurons and their activity}

During an initial attempt to display the distribution of neurons in the premotor cortex, it became apparent that number of taskrelated neurons as well as the magnitude of their activity changes depend on the site of recording. Therefore, there was a need to display the regional distribution of both the number of neurons recorded and the magnitude of their activity changes. This was achieved by summing the magnitude of premovement activity changes (absolute value of the discharge frequency expressed as
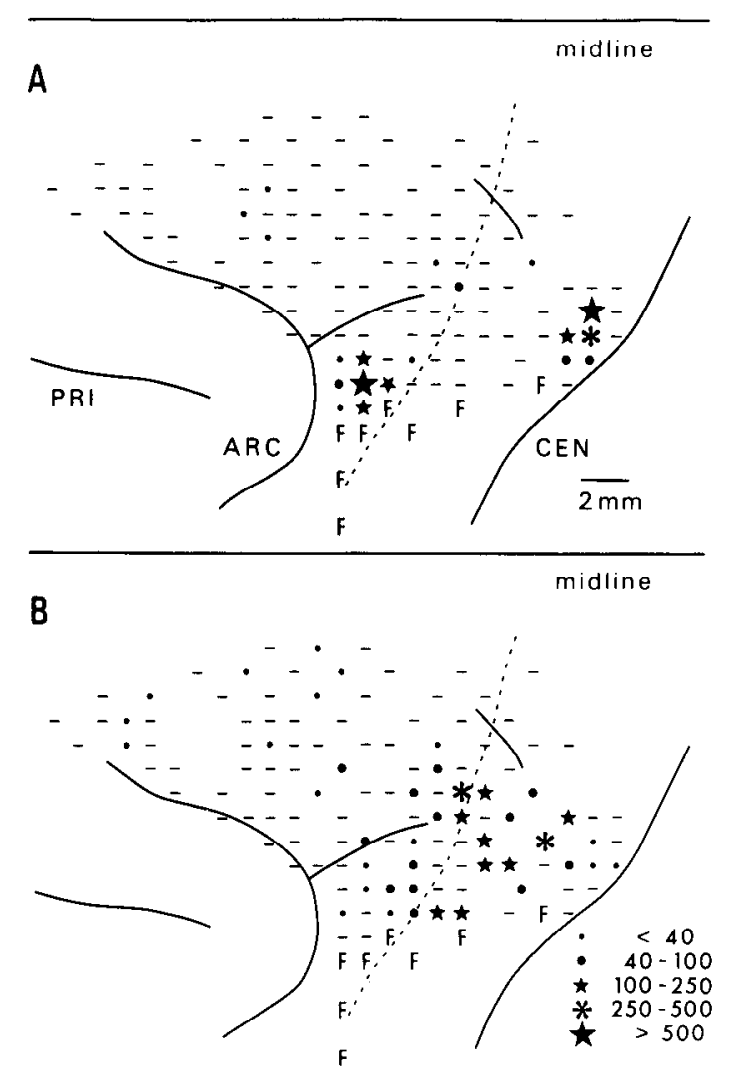

Figure 4. Surface maps of the frontal cortex indicating the distribution of neuronal activity related to the distal $(A)$ and proximal $(B)$ forelimb movements recorded in monkey 1 . Premovement discharge activity (expressed as imp/sec) of neurons recorded at each site was summed and displayed using symbols indicated at bottom right. Short horizontal bars indicate the sites where neither distal nor proximal neurons were recorded. $F$ represents the sites where neurons related to face or mouth movements were recorded. Abbreviations, see Figure 1. The interrupted line indicates the cytoarchitectonic border determined as explained in the text and in Figure 1.

imp/sec during $320 \mathrm{msec}$ prior to the movement onset minus the background discharge frequency) of all task-related neurons obtained at each recording site. For the purpose of display, recording sites were reconstructed in such a way that the cortex was divided into blocks perpendicular to the cortical surface, each $1 \times 1 \mathrm{~mm}$. The magnitudes of the activity changes of neurons belonging to each block were summed and displayed on the cortical surface. The distributions of the summed magnitudes of activity changes thus obtained from monkeys 1 and 3 are displayed in Figures 4 and 5. The magnitudes for distal neurons are displayed in Figures $4 A$ and $5 A$, and those for proximal neurons in Figures $4 B$ and $5 B$, using the symbols indicated at the bottom right. The activity of neurons related to both distal and proximal movements is not plotted on these maps.

PM distal neurons were found mainly in a restricted portion of PM near the genu of the arcuate sulcus. A smaller number of distal neurons with a lower magnitude of activity was also found more medially. Further rostrally, in the area medial to the superior limb of the arcuate sulcus, only a small number of distal neurons was found in monkey 1 (Fig. $4 A$ ) and none was in monkey 3 (Fig. $5 A$ ). Immediately lateral to the sites where distal neurons were recorded in the postarcuate region of PM, neurons were found to be related to licking, chewing, jaw opening, or jaw closing and were therefore categorized as orofacial neurons ( $\mathrm{F}$ in the figures). PM proximal neurons were located more diffusely than distal PM neurons. They were distributed in the postarcuate area posteromedial to the focus of PM distal 

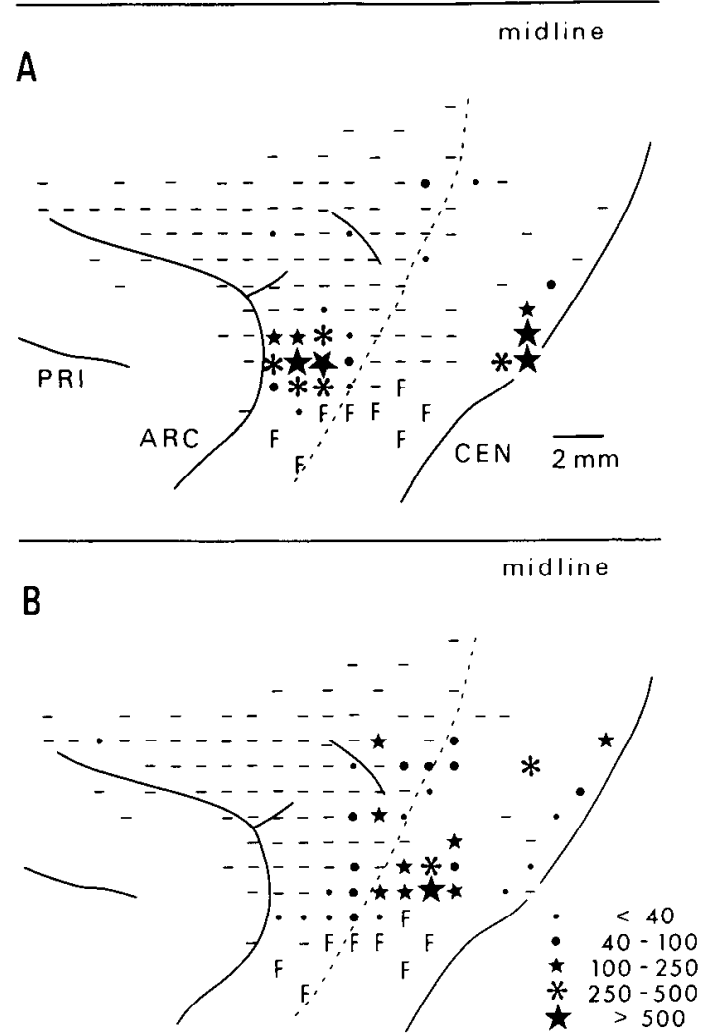

Figure 5. Surface maps of the frontal cortex indicating the distribution of neuronal activity related to the distal $(A)$ and proximal $(B)$ forelimb movements recorded in monkey 3 . Format for the display is the same as in Figure 4.

neurons and also at a region around the precentral sulcus. At all penetration sites in PM, neuronal activity was surveyed throughout its entire depth. This was not true in MI. Some MI penetrations (close to the central sulcus) were terminated before recording all task-related neurons. Owing to this limited sampling, the number of task-related MI neurons (especially distal neurons) was small. Thus, Figures 4 and 5 display the distribution and magnitude of MI activity of neurons related in an incomplete sample. Distal neurons in MI were located in an area close to the central sulcus. MI proximal neurons were distributed more rostrally than distal neurons, and their distribution sites were continuous with those of PM proximal neurons. In monkey 1 , both distal and proximal neurons were also recorded in the supplementary motor area (see Tanji and Kurata, 1979), and they are thus excluded from Figure 4. Neuronal activity was not explored intensively in the supplementary motor area in monkeys 2 and 3.

\section{Response properties of PM neurons to different modalities of movement-triggering signals}

One hundred ninety neurons related to the key-press movement (including distal neurons and mixed type neurons) were recorded from the three monkeys long enough to study quantitatively. Four types of activity change in relation to the key press were observed. The first type was time-locked to the onset of the movement rather than to the onset of the movement-triggering signals (exemplified in Fig. 6A). The second type was timelocked more to the signal onset (Fig. 6B). The third type was biphasic, the first phase being time-locked to signal onset and the second phase time-locked to movement onset (Fig. 6C). In order to categorize the activity changes to these three types, the following analysis was made: The time of occurrence of the first change of activity (the onset of premovement activity increase

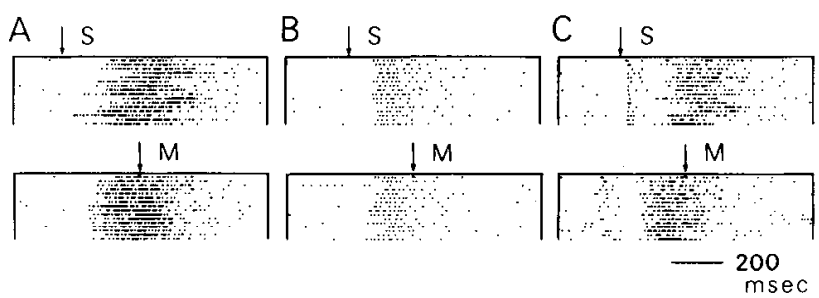

Figure 6. Discharge of PM neurons exhibiting three types of activity in relation to the key-press movement. Arrows labeled with $S$ indicate the onset of trigger signals (visual, auditory, and vibrotactile signals in $A, B$, and $C$, respectively). Arrows labeled with $M$ indicate movement onset. In neuron $A$, the activity is time-locked to movement onset, but in neuron $B$ the activity is time-locked to signal onset. In neuron $C$ the activity is biphasic, exhibiting both signal- and movement-locked activity.

or decrease) was measured for individual trials. In neurons failing to exhibit abrupt activity changes, the measurement was not possible simply by observing the premovement discharge pattern. For these neurons the determination of the onset of the activity changes in each trial was made by utilizing the Kolmogorov-Smirnov test. We used the method described by Mano and Yamamoto (1979) to make this determination and took the 0.05 level to be statistically significant. The interval between the appearance of the movement-triggering sensory signal and the onset of the activity changes was defined as the neuronal response time. The interval between the onset of the activity changes and the movement onset was defined as the lead time. A coefficient of variation $(\mathrm{CV}=\mathrm{SD} /$ mean $\times 100)$ was calculated for the response time and lead time for each unit. If the $\mathrm{CV}$ for the response time was less than $20 \%$, then the unit was classed as signal-locked. If the CV for the lead time was less than $20 \%$, then the unit was classed as movement-locked. If both CVs exceeded $20 \%$, then the activity was categorized as "indeterminate." No PM neuron with monophasic activity changes (e.g., Figs. $6 A$ and $B$ ) was categorized as both movement- and signal-locked. Some neurons showing biphasic activity changes were categorized as both signal-locked and movement-locked. The relative frequencies of these four types of activity changes are displayed in Table 1 . The bottom row of Table 1 indicates that the movement-locked activity was more frequent in PM than the signal-locked activity. Further, the somatosensory signals gave rise to the signal-locked activity more often than either the visual or auditory signal.

Subsequent analysis compared quantitatively the magnitudes of premovement aclivity triggered by each of the sensory signals. For this purpose, the number of discharges during the $320 \mathrm{msec}$ period preceding movement onset was counted in individual trials. The data obtained from 16 consecutive trials responding to the signal of one modality (e.g., visual) were compared with the data obtained in 16 consecutive trials with another signal (e.g., auditory). In 123 neurons (65\%), the magnitudes were similar regardless of the modality of the trigger signals. How-

Table 1. Activity patterns of PM neurons to different modalities of movement-triggering signals ${ }^{a}$

\begin{tabular}{lcccc} 
& $\begin{array}{l}\text { Movement- } \\
\text { locked }\end{array}$ & $\begin{array}{l}\text { Signal- } \\
\text { locked }\end{array}$ & Biphasic & $\begin{array}{c}\text { Indeter- } \\
\text { minate }\end{array}$ \\
\hline Visual & 66 & 8 & 8 & 88 \\
Auditory & 55 & 15 & 8 & 71 \\
Somatic & 38 & 21 & 22 & 59 \\
Total & 159 & 44 & 38 & 218 \\
\hline
\end{tabular}

${ }^{a}$ The total number of responses in each row is different because some cells possessed selective responses to sensory signals of one or two modalities. 

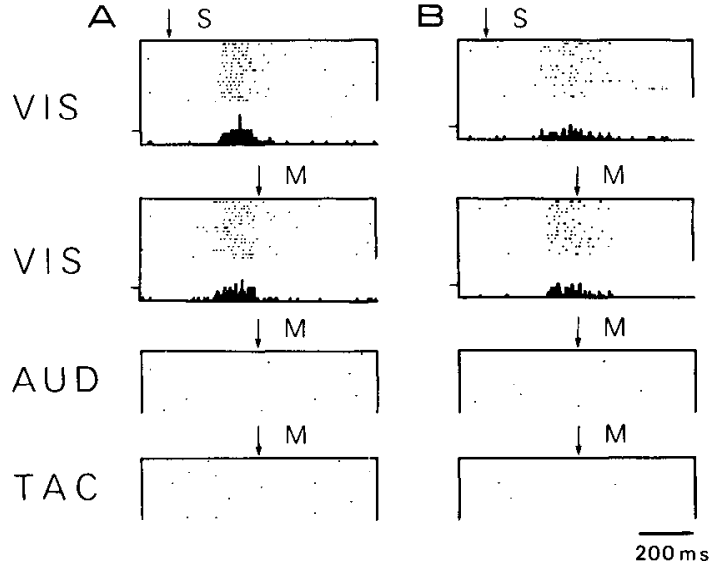

Figure 7. Discharge of two PM neurons active selectively prior to the key-press movement triggered by the visual, but not by the auditory or vibrotactile signal. The discharge activity is time-locked better to the visual signal in neuron $A$, but to the movement onset in neuron $B$. Tick marks on ordinates of histograms in this figure and Figure 8 denote 40 $\mathrm{imp} / \mathrm{sec}$.

ever, in the remaining 67 neurons $(35 \%)$, the magnitudes were significantly different ( $p<0.001 ; t$ test, two-tailed) depending on the modality of the signals (Table 2). Examples of such selective responses are shown in Figures 7 and 8 . Twenty-eight neurons $(15 \%)$ more actively followed the visual signal than either the auditory or tactile signals. Of these 28 neurons having a selective or preferential relation to the visual signal, three exhibited signal-locked activity (Fig. $7 A$ ) and five exhibited movement-locked activity (Fig. $7 B$ ).

A number of other neurons showed a preference for either the tactile or auditory signals. The neuron shown in Figure 8, for instance, was active when the movement was triggered by tactile, but not by visual or auditory, signals. Eleven neurons exhibited preferential activity for the somatosensory signals ("Somatic" in Table 2). Of these, four neurons showed signallocked activity (Fig. 8), three neurons had biphasic activity, and two others showed movement-locked activity. Selective activity to the auditory signal was less frequent (five neurons). In two of these, activity was signal-locked. In another 23 PM neurons the magnitudes were greater in response to the two out of the three signals. The combinations of the two signals with which these PM neurons were preferentially active are indicated in Table 2. The selective or preferential premovement neuronal activity was obtained despite the observations in EMG recordings (confirmed by quantitative analyses) that the magnitude of muscle activity in association with the key-press movement was virtually identical irrespective of the modality of the trigger signals. No MI neuron exhibited preferential premovement activity to any particular signal or activity time-locked with either visual or auditory signal.

Table 2. Responsiveness of PM neurons to three different movementtriggering signals

$\begin{array}{lc}\text { Nondifferential } & 123(65 \%) \\ \text { Differential } & 67(35 \%) \\ \text { Visual (V) } & 28 \\ \text { Auditory (A) } & 5 \\ \text { Somatic (S) } & 11 \\ \text { V and A } & 17 \\ \text { A and S } & 4 \\ \text { S and V } & 2\end{array}$

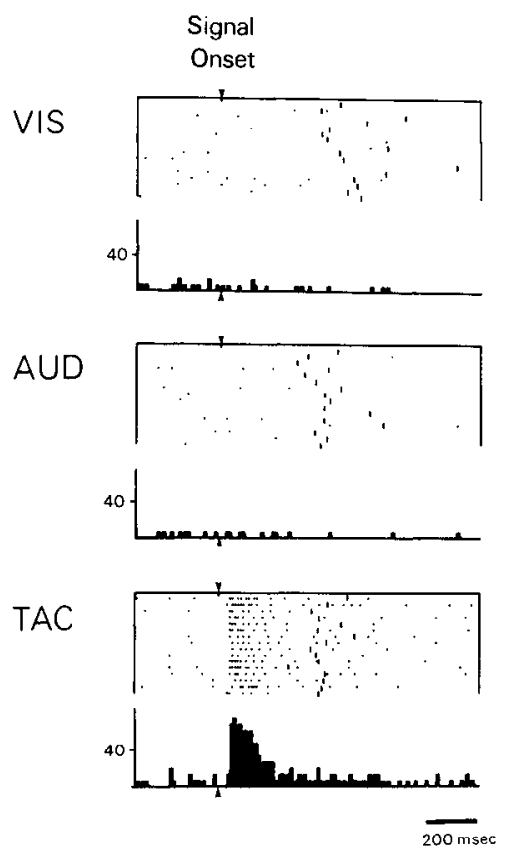

Figure 8. Activity of a PM neuron exhibiting selective response to the vibrotactile trigger signal. Short vertical bars in each line indicate onset of the key-press movement. The neuronal response was time-locked to the onset of the somatosensory signal rather than to the onset of the movement. This neuron did not respond to the vibrotactile stimulus if it did not serve as a movement trigger, nor did it respond to such natural somatosensory stimuli as stroking hairs, brushing, or tapping on limbs.

Response times (the intervals between the signal onset and the onset of neuronal responses) of PM neurons to three different signals were $118 \pm 65,105 \pm 61$, and $69+37 \mathrm{msec}$ following the visual, auditory, and tactile signals, respectively. The response times of PM neurons to the visual and auditory signals were shorter than those of MI neurons $(p<0.001$; see Table 3 ), but there was no statistically significant difference in the response time to the tactile signal in PM and MI neurons. The mean somatosensory response time was, however, $8 \mathrm{msec}$ shorter in MI than in PM. Since sensory signal-locked response to the visual and auditory signal was observed only in PM and not in MI neurons, and the signal-locked response to the tactile signal was more frequent in MI than in PM, the data obtained from neurons possessing signal-locked responses were treated separately in the subsequent analysis and in Table 3 . (The biphasic type of response is included in the signal-locked response.) Even if the signal-locked responses were excluded from the analysis ("Others" in Table 3), the difference in the response times of PM and MI neurons was still significant $(p<0.005)$. The lead times (the intervals between the onset of the neuronal response and the movement onset) were similarly analyzed. Here again, the data obtained from signal-locked responses were treated separately (Table 3 ).

Responsiveness of PM neurons to sensory stimuli other than the signals triggering a movement was tested on 48 PM neurons selected on the basis of apparent response to the movementtriggering signals. None of them responded to a flash of light. The neuronal response to the LED was observed only when it served as the movement trigger, and no response was evoked even in the PM neurons showing selective activity to the visual signal if the same LED was turned on when the monkey was not attending to the task or if it was turned on shortly after the monkey received a reward. Four PM neurons (out of 24 neurons tested) responded to such auditory stimuli as experimenter's whistling, clapping hands, or tapping on a table. These four cells also responded to the $1 \mathrm{kHz}$ tone, even when it was irrelevant 
Table 3. Neuronal response times and premovement lead times of PM and MI in relation to three different triggering signals ${ }^{a}$

\begin{tabular}{|c|c|c|c|c|c|c|}
\hline & \multicolumn{3}{|c|}{ Response times } & \multicolumn{3}{|l|}{ Lead times } \\
\hline & Visual & Auditory & Somatic & Visual & Auditory & Somatic \\
\hline \multicolumn{7}{|l|}{ PM } \\
\hline Total & $118 \pm 65$ & $105 \pm 61$ & $69 \pm 37$ & $142 \pm 66$ & $151 \pm 71$ & $160 \pm 83$ \\
\hline Signal-locked & $81 \pm 16$ & $72 \pm 14$ & $48 \pm 14$ & $191 \pm 53$ & $193 \pm 62$ & $182 \pm 49$ \\
\hline Others & $122 \pm 40$ & $111 \pm 34$ & $78 \pm 29$ & $137 \pm 49$ & $142 \pm 50$ & $150 \pm 57$ \\
\hline \multicolumn{7}{|l|}{ MI } \\
\hline Total & $161 \pm 71$ & $142 \pm 59$ & $61 \pm 34$ & $87 \pm 34$ & $89 \pm 30$ & $103 \pm 47$ \\
\hline Signal-locked & - & - & $41 \pm 11$ & - & - & $125 \pm 41$ \\
\hline Others & $161 \pm 71$ & $142 \pm 59$ & $70 \pm 28$ & $87 \pm 34$ & $89 \pm 30$ & $93 \pm 28$ \\
\hline
\end{tabular}

${ }^{a}$ Values are means \pm SD (in msec).

to the task performance. Eight neurons (out of 19 tested) responded to somatosensory natural stimuli, such as tapping or brushing the forelimb, and also responded to the $40 \mathrm{~Hz}$ vibrotactile signal even when irrelevant to the task performance. Although the somatosensory response properties were studied intensively in only five of these neurons, it was noted that these receptive fields were generally broad, including a considerable part of contralateral forelimb or part of trunk and forelimb; in the other neurons, they included a part of the hindlimb as well as forelimb. Bilateral somatosensory receptive fields were detected in two neurons. The response magnitude was variable and often attenuated when a natural stimulus was repeated.

Eight distal neurons were tested for their relation to spontaneously performed movements by temporarily interrupting the task performance. All of them were active when the animal pressed the key "spontaneously," not triggered by any sensory signals. Four of the eight neurons belonged to the indeterminant category.

Although PM neurons with preferential responses to a particular signal were often recorded in succession during penetration of the cortex, no trends for preferential distribution of modality-specific sensory responses were detectable anywhere in PM, and the density of such selective neurons seems to be proportional to the density of distal neurons in general. No attempt was made to detcrminc the laminar distribution of particular response types.

\section{Discussion}

Premotor cortex as defined in the present study

Since no general agreement has yet been established as to the subdivision of the frontal agranular cortex, we chose to define a motor field, the premotor cortex, on the basis of the cytoarchitectonics and electrophysiological findings (utilizing intracortical microstimulation, ICMS) according to the proposal made by Weinrich and Wise (1982). However, since the transition of the density of larger pyramidal cells in layer $\mathrm{V}$ is gradual and effects of ICMS can vary greatly with differences in stimulus intensity, the definition used in the present study must be viewed as tentative. With more intense stimulus currents than utilized in the present study $(50 \mu \mathrm{A})$, it is entirely possible that limb movements could be evoked from broader cortical areas.

\section{Topography}

The presence of neurons specifically related to distal limb movements suggests that PM is not solely specialized for proximal and/or axial motor control. On the basis of the present study, we conclude that PM, as defined here, plays a significant role in the neural control of distal as well as proximal limb move- ments (see introduction). It should be mentioned that this definition and use of the term PM do not imply that the whole PM area is functionally uniform. In fact, evidence has been accumulating that the PM has separate subregions having different functional roles (for review, see Humphrey, 1979; see also Godschalk et al., 1984; Muakkassa and Strick, 1979; Wise, 1985), and one or more subregions may indeed be specialized for proximal motor control. Combining the present data for both distal and proximal neurons, the cortical distribution of forelimb taskrelated PM neurons corresponds well with that of recent reports (Godschalk et al., 1981; Weinrich et al,, 1984), in which monkeys performed a motor task involving both proximal and distal limb movements. The activity of PM distal neurons was focused in the postarcuate area close to the genu of the arcuate sulcus. The activity of proximal neurons was distributed slightly posteromedially to the focus of distal neurons in postarcuate region, though there was considerable overlap in these two populations of neurons. These data are comparable with those by Brinkman and Porter (1983) and suggest a topographically organized subdivision of PM. Since in the present experiment the key-press movement was triggered by sensory signals and the rotation movement was self-paced, there is a possibility that the difference of distribution may be due to different localization of PM neurons related to signal-triggered versus self-paced movements. However, the distal neurons were also active in relation to self-paced key-press movement (though the number of neurons tested for the self-paced movement was not large); therefore, it is more likely that PM neurons related to distal and proximal forelimb movements are distributed differently. The possibility of somatotopic organization of the PM was proposed by Muakkassa and Strick (1979), who demonstrated that the corticocortical projection from PM to MI is topographically organized. A recent report by Godschalk et al. (1984) extended the knowledge of the PM corticocortical projection by both tracer-injection and electrophysiological methods. They reported that the postarcuate projection to MI is arranged in a parallel fashion: The lateral part of the postarcuate area projects to the lateral part of MI, and the posteromedial part of the postarcuate area projects to posteromedial part of MI. These reports are in accord with the present findings on movementrelated neuronal activity, and together they suggest a somatotopic organization: In the lateralmost part of the postarcuate area lie neurons related to face or mouth movements; in the area around the genu of the arcuate sulcus lie neurons related to distal forelimb neurons; and more posteromedially lie proximal forelimb neurons. In the area medial to the upper limb of the arcuate sulcus, only a small number of proximal neurons was obtained in this study and only negligible activity was found in association with the distal forelimb movement. This 
negative finding is consistent with the view proposed by Humphrey (1979), that this part of the agranular frontal cortex dorsomedial to the arcuate sulcus, in conjunction with other frontal cortical areas, may exert tonic position or postural effects and produce coordinated orienting movements of numerous body parts, rather than generating direct reaching movements. It should be pointed out, however, that in the present study the neuronal activity was observed in relation to simple movements; thus, there remains the possibility that in association with morc complex movements or with complex sequences of movements, the topography may appear differently organized.

\section{Modality specificity of movement related units}

Twenty-eight PM neurons (15\%) were active preferentially prior to visually triggered key-press movement in this experiment. This finding is consistent with the idea that PM has a role in visually guided movement. However, a large number of PM neurons $(65 \%)$ were active prior to the movement triggered by all of three different signals, and furthermore, 20 neurons were active preferentially with somatosensory and/or auditory signals. These findings indicate that the visual signal does not seem to be the sole source of sensory input to activate PM neurons in association with a simple triggered movement. Responsiveness of PM neurons to the auditory signal was observed recently (Weinrich and Wise, 1982), when an auditory cue was used as an instruction for an impending movement. Somatosensory responses of PM neurons were reported in anesthetized and curarized monkeys (Rizzolatti et al., 1981a). The present findings indicate that auditory and somatosensory inputs are no less potent than visual stimuli in activating PM neurons when these stimuli are used as a movement trigger. This view is consistent with anatomical studies that indicate an input to PM from sensory association areas (Chavis and Pandya, 1976; Godschalk et al., 1984; Pandya and Kuypers, 1969; Petrides and Pandya, 1984). The signal-induced PM activity may influence MI neurons by corticocortical connections and may, in part, give rise to MI movement-related activity. The response and lead times of PM and MI neurons (Table 3) are consistent with this possibility, though in no way proving the causal relation between the two areas. Weinrich et al. (1984) also found that PM neuronal activity preceded that of $\mathrm{MI}$ in relation to arm movements. However, since there was little attempt in the previous studies to identify the axonal destination of movement-related PM neurons, there was no way of knowing if movement-related neurons send direct outputs to MI or descending outputs to brain stem and/or spinal cord. The present data show that at least some of the movement-related cells are corticofugal and have axons in the pyramidal tract.

\section{Comparison of $P M, M I$, and $S M A$}

The PM neurons showed activity before either distal or proximal forelimb movements as well as the MI neurons. However, PM neurons had different properties from MI. These properties included (1) less movement-related activity modulation in PM neurons than that in MI neurons; (2) the presence of selective or preferential responses to sensory signals of different modalities triggering an identical movement in PM, but not in MI; (3) shorter response times to visual and auditory signals in PM compared to MI neurons; (4) the presence of activity timelocked to visual or auditory signals in PM but not in MI (see also Lamarre et al., 1983). The third and fourth findings are in accord with those reported by Kubota and Hamada (1978). They reported responses of PM neurons to a visual movement-triggering signal with latency of as short as $60 \mathrm{msec}$ that were coupled in time to the visual signal. The latencies of movementcoupled responses were $80-150 \mathrm{msec}$. They also reported that vcry fcw (10-15\%) PM ncurons displayed a discharge pattcrn that correlated significantly with the parameters of wrist move- ments (velocity and acceleration), in contrast to PTNs in MI.

It is of interest that a number of SMA neurons exhibit activity properties in common with those in PM. In addition to the four properties mentioned above, it was reported (Brinkman and Porter, 1983; Wise and Tanji, 1981) that somatosensory responses in SMA are harder to obtain and smaller in magnitude than in MI. Also, Tanji and Kurata (1982) reported that few, if any, of the SMA neurons showing responses to visual or auditory movement-triggering signals were passively activated by the same sensory signal when it was irrelevant to the task. This behavior is comparable to the PM neurons described in this report. Together, these findings suggest that PM and SMA may play a similar role in sensorally triggered movement. Further, some neurons in both PM and SMA are modulated in relation to self-initiated movements, suggesting a similarity in their role for these movements as well. In contrast to this view, it has been proposed that PM plays an important role in sensorially guided movement and that SMA may be more important in self-initiated movement (Evarts and Wise, 1984; Goldberg, in press; Rizolatti et al., 1983; Roland et al., 1983; Wise and Mauritz, 1985). In order to resolve this question, it would be very interesting to analyze the neuronal activity of PM and SMA in a behavioral paradigm specifically designed to compare activity in association with sensorially guided, as opposed to self-initiated, movement.

\section{References}

Bonin, G. von, and P. Bailey (1947) The Neocortex of Macaca mulatta, University of Illinois Press, Urbana, IL.

Brinkman, C., and R. Porter (1983) Supplementary motor area and premotor area of monkey cerebral cortex: Functional organization and activities of single neurons during performance of a learned movement. In Motor Control Mechanisms in Health and Disease, J. E. Desmedt, ed., pp. 393-420, Raven, New York.

Brodmann, K. (1909) Vergleichende Lokalizationlehre der Grosshirnrinde in ihren Prinzipien Dargestellt auf Grund des Zellenbaues, J. A. Barth, Leipzig.

Chavis, D. A., and D. N. Pandya (1976) Further observations on corticofrontal connections in the rhesus monkey. Brain Res. 117:369386.

Evarts, E. V. (1968) A technique for recording activity of subcortical neurons in moving animals. Electroencephalogr. Clin. Neurophysiol. 24: $83-86$.

Evarts, E. V., and S. P. Wise (1984) Basal ganglia outputs and motor control. In Functions of the Basal Ganglia, Ciba Foundation Symposium 107, pp. 83-96, Pitman, London.

Freund, H.-J., and H. Hummelsheim (1984) Premotor cortex in man: Evidence for innervation of proximal limb muscles. Exp. Brain Res. 53: 479-482.

Fulton, J. F. (1934) Forced grasping in relation to the syndrome of the premotor area. Arch. Neurol. Psychiatry 31: 221-235.

Godschalk, M., R. N. Lemon, H. G. J. M. Kuypers, and K. Ronday (1984) Cortical afferents and efferents of monkey postarcuate area. An anatomical and electrophysiological study. Exp. Brain Res. 56: $410-424$.

Godschalk, M., R. N. Lemon, H. G. T. Nijs, and H. G. J. M. Kuypers (1981) Behavior of neurons in monkey periarcuate and precentral cortex before and during visually guided arm and hand movements. Exp. Brain Res. 44: 113-116.

Goldberg, G. (in press) Supplementary motor area structure and function: Review and hypothesis. Behav. Brain Sci.

Haaxma, R., and H. G. J. M. Kuypers (1975) Intrahemispheric cortical connexions and visual guidance of hand and finger movements in the rhesus monkey. Brain 98: 239-260.

Halsband, U., and R. Passingham (1982) The role of premotor and parietal cortex in the direction of action. Brain Res. 240:368-372.

Humphrey, D. R. (1979) On the cortical control of visually directed reaching: Contributions by nonprecentral motor arcas. In Posture and Movement, R. E. Talbott and D. R. Humphrey, eds., pp. 51-112, Raven, New York.

Kubota, K., and I. Hamada (1978) Visual tracking and neuron activity in the post-arcuate area in monkeys. J. Physiol. (Paris) 74: 297-313. 
Kuypers, H. G. J. M., and J. Brinkman (1970) Precentral projections to different parts of the spinal intermediate zone in the rhesus monkey. Brain Res. 24: 29-48.

Kuypers, H. G. J. M., and D. G. Lawrence (1967) Cortical projections to the red nucleus and the brain-stem in the rhesus monkey. Brain Res. 4: 151-188.

Lamarre, Y., L. Busby, and G. Spidalieri (1983) Fast ballistic arm movements triggered by visual, auditory, and somesthetic stimuli in the monkey. I. Activity of precentral cortical neurons. J. Neurophysiol. 50: 1343-1358.

Mano, M., and K. Yamamoto (1979) Kolmogorov-Smirnov test for the onset time determination of modulation in discharge rate. In Integrative Control Functions of the Brain, Vol. 2, M. Ito, ed., pp. 146-148, Kodansha, Tokyo.

Matsumura, M., and K. Kubota (1979) Cortical projection to handarm motor area from post-arcuate in macaque monkeys: A histological study of retrograde transport of horseradish peroxidase. Neurosci. Lett. 11: 241-246.

Moll, L., and H. G. J. M. Kuypers (1977) Premotor cortical ablations in monkeys: Contralateral changes in visually guided reaching behavior. Science 98: 317-319.

Muakkassa, K. F., and P. L. Strick (1979) Frontal lobe inputs to primate motor cortex: Evidence for four somatotopically organized 'premotor' areas. Brain Res. 177: 176-182.

Pandya, D. N., and H. G. J. M. Kuypers (1969) Corticocortical connections in the rhesus monkey. Brain Res. 13: 13-36.

Petrides, M., and D. N. Pandya (1984) Projection to the frontal cortex from the posterior parietal region in the rhesus monkey. J. Comp. Neurol. 228: 105-116

Rizzolatti, G., M. Matelli, and G. Pavasi (1983) Deficits in attention and movement following the removal of postarcuate (area 6) and prearcuate (area 8) in macaque monkeys. Brain 106: 655-673.

Rizzolatti, G., C. Scandolara, M. Matelli, and M. Gentilucci (1981a) Afferent properties of periarcuate neurons in macaque monkeys. 1. Somatosensory responses. Behav. Brain Res. 2: 125-146.

Rizzolatti, G., C. Scandolara, M. Matelli, and M. Gentilucci (1981b) Afferent properties of periarcuate neurons in macaque monkeys. 2. Visual responses. Behav. Brain Res. 2: 147-163.
Roland, P. E., B. Larsen, N. A. Lassen, and E. Skinhoj (1983) Supplementary motor area and other cortical areas in organization of voluntary movements in man. J. Neurophysiol. 43: 118-136.

Sessle, B. J., and M. Wiesendanger (1982) Structural and functional definition of the motor cortex in the monkey (Macaca fascicularis). I. Physiol. (Lond.) 323: 245-265.

Tanji, J., and K. Kurata (1979) Neuronal activity in the cortical supplementary motor area related with distal and proximal forelimb movements. Neurosci. Lett. 12: 201-206.

Tanji, J., and K. Kurata (1982) Comparison of movement-related activity in two motor areas of primates. J. Neurophysiol. 48: 633653.

Vogt, O., and C. Vogt (1919) Ergebnisse unserer Hirnforschung. J. Psychol. Neurol. (Leipzig) 25: 277-462.

Weinrich, M., and S. P. Wise (1982) The premotor cortex of the monkey. J. Neurosci. 2: 1329-1345.

Weinrich, M., S. P. Wise, and K.-H. Mauritz (1984) A neurophysiological study of the premotor cortex in the rhesus monkey. Brain 107: $385-414$

Wiesendanger, M. (1981) Organization of secondary motor areas of cerebral cortex. In Handbook of Physiology. Section I: The Nervous System. Vol. II: Motor Control, Pt. 2, V. B. Brooks, ed., pp. 11211147, American Physiological Society, Bethesda, MD.

Wise, S. P. (1985) The primate premotor cortex: Past, present and preparatory. Annu. Rev. Neurosci. 8: 1-19.

Wise, S. P., and K.-HI. Mauritz (1985) Set-related neuronal activity in the premotor cortex of rhesus monkeys: Effects of changes in motor set. Proc. R. Soc. Lond. [Biol.] 223: 331-354.

Wise, S. P., and J. Tanji (1981) Supplementary and precentral motor cortex: Contrast in responsiveness to peripheral input in the hindlimb area of the unanesthetized monkey. J. Comp. Neurol. 195: 433-451.

Woolsey, C. N., P. H. Settlage, D. R. Meyer, W. Spencer, T. P. Hamuy, and A. M. Travis (1952) Patterns of localization in precentral and 'supplementary' motor areas and their relation to the concept of a premotor area. Res. Publ. Assoc. Res. Nerv. Ment. Dis. 30: 238-264. 